

\title{
Effects of Post Exercise Meal Timing on Glucose Homeostasis in Trained Obese and Non-Obese Rats
}

\author{
Saad Kamal Taha, Merhan Mamdouh Ragy*and Ahmed Moustafa Mahmoud** \\ Departments of Physiology, Al-Azhar, El-Minia* and Sohag** Faculties of Medicine
}

Received: 20 March 2015

Accepted: 26 April 2015

Available online: 1 May 2015

\section{Keywords}

- Muscular exercise

- Obesity

- Training

- Metabolism

\begin{abstract}
Background: Obesity is a risk factor for obesity-related disorders such as type 2 diabetes mellitus. That obesity could hinder the individual work capacity and the cost for managing obesity complications. Aim of Work: was to study the effect of diet induced obesity and swimming on the plasma glucose, insulin, insulin resistance, body mass index (BMI), muscle mass and muscle glycogen, and fat tissue weight according to meal timing. Materials and Methods: Sixty Sprague dawely adult rats were randomized into two equal groups control group and obese group. The first was exposed for induction of obesity by hyper caloric diet and the other completes the standard one. At the end of the 12th week of age, rats started the exercise program for three months. The immediate group receives their meals immediately after exercise and the delayed one receive their meals three hours later. Then after receiving their meal, free access to food was allowed. Samples of blood were taken from all animals at the beginning and at the end of experiment and examined for plasma glucose and plasma insulin. Insulin resistance was estimated by HOMA-IR formula. Results: Immediate feeding after the exercise bout produced greater increase in muscle bulk and muscle glycogen, with decreased fat tissue weight in comparison with that of the corresponding delayed feeding either on obese and control groups Conclusion: Immediate meal timing was a powerful strategy to improve glucose homeostasis. Data of the present study showed that muscle bulk was greater with lower fat tissue weight in the immediate feeding group versus the delayed one.
\end{abstract}

Corresponding author: Ahmed Mostafa, Department of Medical Physiology, Departments of Physiology, Sohag Faculty of Medicine, Sohag, Egypt. phone: +201060423663, e-mail: ahmed_physiology@yahoo.com 


\section{INTRODUCTION}

The incidence of obesity and its association with co morbidities is dramatically increased worldwide. Its prevalence is also related to environmental factors, especially diet and reduced physical activity (1). The incidence of obesity between Egyptians aged 15 to 100 year is $22 \%$ in males and $48 \%$ in females (2). Since the adverse consequences of obesity on health are directly related to excess body fat, the ideal method of classification of obesity should be based on direct measurement of fatness, which is expressed as percentage body fat (PBF), but this may not be easily attainable. In the clinical environment, techniques such as body mass index BMI, waist circumference, and waist circumference over hip circumference ratio were used extensively. Although, these methods are less accurate, they are satisfactory in identifying the individuals who are at risk of, weight-related, health problems (3). Regular exercise combined with dietary intervention is more successful than pharmacological intervention in the treatment and prevention of obesity (4). Change dietary habits can help weight management (5). Timing of food intake itself may have a significant role in obesity as the time of feeding, particularly for high-energy content meals, may be decisive, and changes in this timing could have metabolic consequences for the development of obesity and for weight loss (6). But, the effect of Timing of food intake post exercise on fat mass and glucose homeostasis in obesity is unclear.

This study was conduct on a trial to
1- Demonstrate the effect of post exercise meal timing on BMI, fat accumulation, gastrocnemius ms mass and its glycogen in lean and obese groups and

2- Answer a question (it is it better to receive meals immediately after exercise or to delay it for three hours later on obese and non-obese adult male albino rats?).

\section{MATERIALS AND METHODS}

Sixty Adult male albino (Sprague Dawely strain) rats, about 4 months old were used throughout the present study. Rats were purchased from the National Research Center, Cairo, Egypt. They were housed at room temperature with natural light/dark cycles in suitable cage. Rats were fed a standard diet of commercial rat chow and tap water and left to acclimatize to environment for two weeks prior to inclusion in the experiment. Rats were randomized into two equal groups. The first was exposed for induction of obesity by high fat diet and the other completes the standard one (7).

Table (1) : Composition of the standard diet of commercial rat chow and the high fat diet

\begin{tabular}{|l|l|}
\hline \multicolumn{1}{|c|}{ The high fat diet } & \multicolumn{1}{|c|}{ The slandered diet } \\
\hline Fat 46\% [corn oil 25.5\%, & Fat 5\% [corn oil] \\
and beef tallow 20.5\%] & Carbohydrates 65\% \\
Carbohydrates 24\% [corn & [corn starch 15\% and \\
starch 6\% and sucrose & sucrose 50\%], \\
$18 \%$ Proteins 20.3\% [casein \\
Proteins 20.3\% [casein & 20\% and DL- \\
$20 \%$ and DL-Methionine & Methionine 3\%], \\
$3 \%$ F & Fiber 5\% \\
Fiber 5\% & Salt mixture 3.7\%, \\
Salt mixture 3.7\%, & Vitamin mixture 1\%). \\
Vitamin mixture 1\%). & \\
\hline
\end{tabular}


Lee index was calculated for each rat according to the following formula:Cube root of body weight (g) X 10/nasoanal length(mm) All rats fed with HFD had a Lee index higher than 0.3 and are considered obese (8).

All rats were weighed and their nose to tail length was measured and body mass index was detected weekly according to the following formula:-

-Body mass index $(\mathrm{BMI})=$ body weight (g)/length2 (cm2).

- Rats started the exercise program for three months. The immediate group receives their meals immediately after exercise and the delayed one receive their meals three hours later. Then after receiving their meal, free access to food was allowed.

- Obese group (30 rats): Divided into three equal subgroups :

a) Obese non-exercised $(\mathrm{ON})$.

b) Obese exercised every other day and take their meal immediately after exercise (OI).

c) Obese exercised every other day and take their meal three hours after exercise (OD).

- Non obese (lean) group (30 rats): Divided into three equal subgroups :

a) Lean non exercised groups $(\mathrm{CN})$.

b) Lean exercised rats exercise every other day and receive their meal immediately after exercise (CI).

c) Lean exercised rats exercise every other day and receive their meal three hours after exercise (CD).

\section{Exercise swimming program}

At the beginning of the program, the aquatically native rats were given the chance to stay in water bath for a short time in few numbers. After becoming familiar with water rats were put in water bath in large numbers and were urged to swim actively all the time. Rats in exercise groups were exercised by swimming $1 \mathrm{hrs} /$ day for 12 weeks. The swimming exercise was performed in plastic barrel $(50 \mathrm{~cm})$ diameter filled with water (50 cm deep) maintained at $32-36^{\circ} \mathrm{C}$. Rats stop swimming one day before decapitation and were left fasting for $12 \mathrm{hrs}$ before decapitation (9).

\section{Collection of blood samples}

Samples of blood were taken from all animals at the beginning by retro orbital route and at the end of experiment by decapitation. Fasting blood samples were withdrawn through the Retro-orbital route using heparinized capillary tubes inserted in medial canthus medial to eye globe. The blood samples were delivered into centrifuge tubes to which anticoagulant were added. They were then centrifuged for 20 minutes and plasma was separated and stored as $-70{ }^{\circ} \mathrm{C}$ until used. These samples were examined for plasma glucose and plasma insulin. Insulin resistance was estimated by HOMA-IR formula.

\section{Measurement of serum fasting glucose, insulin and HOMA-IR index}

Serum insulin was measured by ELISA ACTIVE Insulin Enzyme-Linked Immunosorbent (ELISA) by Glory Science kits (10), serum glucose by enzymatic colorimetric method by Biodiagnostic, Egypt kits (11) and insulin resistance was estimated by HOMA-IR (homeostasis model assessment; IR, insulin resistance), which was 
calculated as (fasting blood glucose $(\mathrm{mmol} / \mathrm{l}) \mathrm{x}$ fasting insulin (mU/l)) / 22.5 (12 ).

\section{Determination of fat mass and Glycogen Content:}

Mesenteric, retroperitoneal and epididymal adipose tissue were immediately removed and weighed. This present work concentrates on visceral fat mainly, because visceral fat is more important than the subcutaneous fat in the development of obesity-induced insulin resistance (13).

The method of glycogen content measurement in muscle was adopted from (14). . It was based on heating of glycogen solution with concentrated sulphuric acid induces dehydration of the former and development of pink color, the intensity of which is proportionate to the glycogen concentration. The intensity of the pink color was read spectrophotometrically at $520 \mathrm{~nm}$.

\section{Statistical Evaluation of Experimental Data:}

Data were presented as means \pm SE (Standard Error). The Student's t-test was utilized to determine if differences existed between the (lean and obese), (trained and sedentary) and (immediate and delayed) animals at the beginning and end of the study. Two-way analysis of variance (ANOVA) followed by a Student Newman-Keuls post hoc analysis was employed to assess the effects of obesity, training and meal timing on the dependent variables. The level of significance accepted when $\mathrm{P}<0.05$.

\section{RESULTS}

After obesity induction, Obese groups (ON, OI and OD) showed significant higher BMI and total fat tissue weight than their corresponding control groups $(\mathrm{CN}, \mathrm{CI}$ and $\mathrm{CD})$ respectively. Trained groups (CI and $\mathrm{CD}$ ) and (OI and $\mathrm{OD}$ ) showed significant lower BMI and fat weight than their corresponding non exercised groups $\mathrm{CN}$ and $\mathrm{ON}$ respectively. Immediate feeding groups $(\mathrm{CI}$ and OI) showed no significant difference in BMI with their corresponding delayed feeding groups (CD and $\mathrm{OD})$ respectively. Immediate feeding groups (CI and OI) showed significant lower fat tissue weight with their corresponding delayed feeding groups (CD and OD) respectively (Fig. $1 \& 2$ ).

Obese groups (ON, OI and OD) showed significant lower gastrocnemius muscle weight with their corresponding control groups $(\mathrm{CN}, \mathrm{CI}$ and $\mathrm{CD}$ ) respectively. Trained groups (CI and CD) and (OI and OD) showed significant higher gastrocnemius muscle weight than their corresponding non exercised groups $\mathrm{CN}$ and $\mathrm{ON}$ respectively. Immediate feeding groups (CI and OI) groups showed significant higher gastrocnemius muscle mass with their corresponding delayed feeding groups (CD and OD) respectively (Figure 3).

Obese groups (ON, OI and OD) showed significant higher muscle glycogen content with their corresponding control groups $(\mathrm{CN}, \mathrm{CI}$ and $\mathrm{CD})$ respectively. Trained groups (CI and $\mathrm{CD}$ ) and (OI and OD) showed significant higher gastrocnemius muscle glycogen content than their corresponding non exercised groups $\mathrm{CN}$ and $\mathrm{ON}$ respectively. Immediate feeding groups (CI and OI) groups showed significant higher gastrocnemius muscle glycogen content with their corresponding delayed feeding groups $(\mathrm{CD}$ and OD) respectively (Figure 4). 
At the start of the training, schedule there was a significant higher plasma glucose between the obese groups (ON, OI and OD) and their corresponding control groups $(\mathrm{CN}, \mathrm{CI}$ and $\mathrm{CD})$. At the end of the exercise schedule, the ON group showed significant higher plasma glucose level than that of the corresponding $\mathrm{CN}$ group but with training the obese exercised groups (OI and OD) showed no significant difference with the corresponding control exercised (CI and $\mathrm{CD}$ ) one. Trained groups (CI and CD) showed no significant difference in plasma glucose level with their corresponding non exercised groups $(\mathrm{CN})$, however OI and OD groups showed significant difference with their corresponding ON group. Immediate feeding groups (CI and OI) showed no significant difference with their corresponding delayed feeding groups (CD and OD) respectively. Trained groups (CI and $\mathrm{CD}$ ) and (OI and OD) groups showed significant lower plasma glucose than their pre-training values (PPCI and PPCD) and (PPOI and PPOD) groups respectively (Figure $5)$.

At the start of the training schedule, there was significant higher plasma insulin between the obese groups (ON, OI and OD) and their corresponding control groups ( $\mathrm{CN}, \mathrm{CI}$ and $\mathrm{CD})$. At the end of the exercise schedule, obese groups (ON, OI and OD) showed significant higher plasma insulin level than their corresponding control groups ( $\mathrm{CN}, \mathrm{CI}$ and $\mathrm{CD}$ ) respectively. Trained obese groups (OI and OD) showed significant lower plasma insulin with their corresponding non exercised groups ON. Immediate feeding groups (CI and OI) groups showed no significant difference in plasma insulin level with their corresponding delayed feeding groups (CD and OD) respectively. Trained groups (OI and OD) groups showed significant lower plasma insulin level with their corresponding pretraining values (Figure 6).

At the beginning of the training schedule, there was a significant higher insulin resistance values between the obese groups (ON,OI and OD) and their corresponding control groups $(\mathrm{CN}, \mathrm{CI}$ and $\mathrm{CD})$. Obese groups (ON, OI and OD) showed significant higher insulin resistance with their corresponding control groups $(\mathrm{CN}, \mathrm{CI}$ and $\mathrm{CD})$ respectively. Trained groups (OI and OD) showed significant lower insulin resistance than their corresponding non exercised groups $\mathrm{ON}$ respectively. However, within the control groups $(\mathrm{CN}, \mathrm{CI}$ and $\mathrm{CD})$, there was no significant difference in insulin resistance. Immediate feeding groups (CI and OI) showed no significant difference in insulin resistance with their corresponding delayed feeding groups (CD and OD) respectively. All trained groups (CI and $\mathrm{CD}$ ) and (OI and $\mathrm{OD})$ showed significant decrease in insulin resistance than their corresponding pertraining values (Figure 7). 




- significant difilerence whe corresponding control group.

* Significant difierence wib the corresponding non exercised group.

- Signilicant difierence will the sane group be bre training.

Fig.(1): Comparative results in BMI between different groups.



Fig.(2): Comparative results in fat tissue mass between different groups.



Fig.(3): Comparative results in gastrocnemius muscle mass between different groups at the end of the study. 




Fig. (4): Comparative results in gastrocnemius muscle glycogen content between different groups at the end of the study.

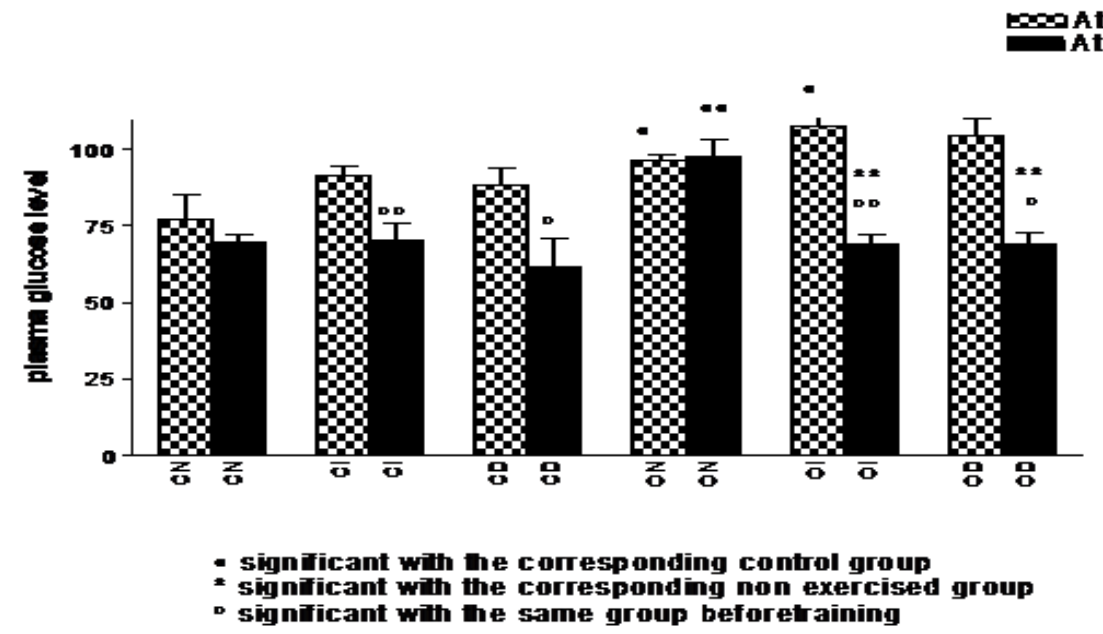

Fig. (5): Difference in plasma glucose level between different groups at the beginning and the end of the study.

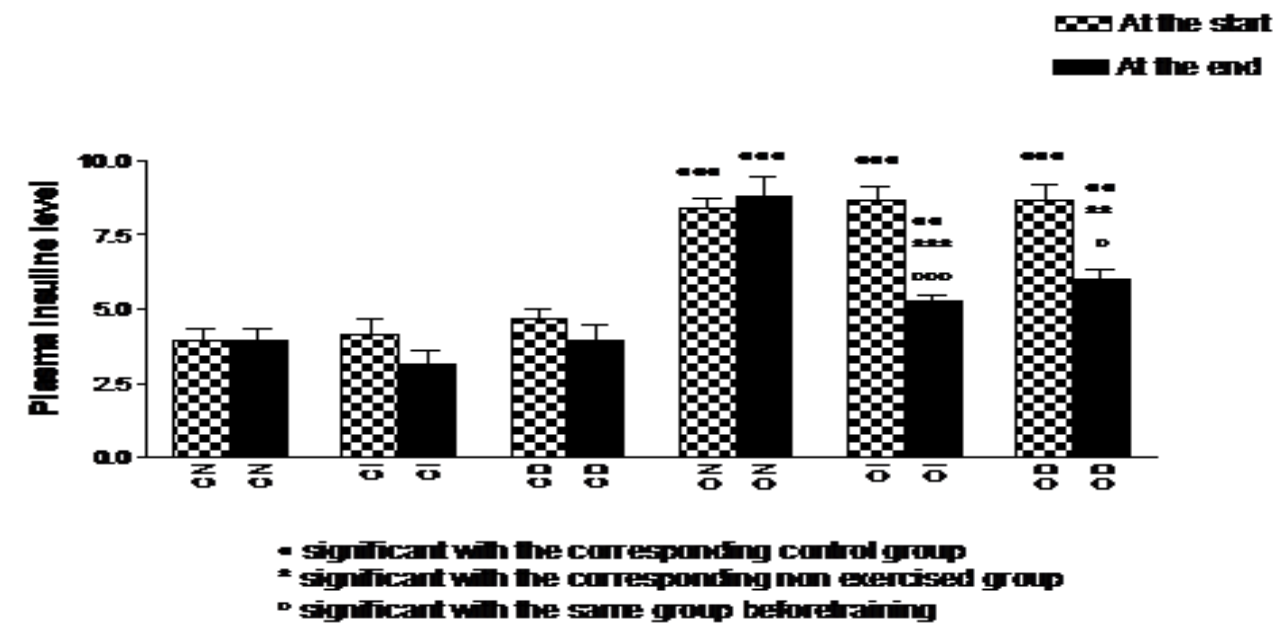

Fig.(6): Difference in plasma insulin level between different groups at the beginning and the end of the study. 


\section{DISCUSSION}

The present work demonstrated higher BMI in the obese rats compared to non obese rats which in agreement with Abdel Malek. (15) and Arnold et al. (16). Also, the final BMI in the trained rats (OI and $\mathrm{OD}, \mathrm{CI}$ and $\mathrm{CD}$ ) was lower than non trained rats which are in line with that reported by previous studies $(14,17)$.

The mechanisms have been suggested for increased BMI by HFD include: (1) high palatability of fat and overconsumption of high caloric diet (18); (2) HFDs lower the rate of oxidation of fatty acids and increase lipogenesis (19).

BMI was still higher in the trained obese (OI and OD) than their corresponding trained control group (CI and CD). This agreed with Pinheiro-Mulder et al. (20), Schroeder et al. (21) and Arnold et al (16). Matsakas et al. (22) explained the decrease in BMI with training (swimming) by the decrease in food intake and body fat component. Endurance exercise induce loss of fat mass by increasing fat oxidation through increasing mitochondrial density, the activity of enzymes involved in $\beta$ oxidation and oxygen delivery to muscle and increases the gene expression and protein content of several fatty acid transporters, which aid in the uptake and delivery of fatty acids to mitochondria (23). Also, adaptation to endurance training increases the energy expenditure helping body weight loss (17). In the present work, immediate feeding (CI and OI) showed non- significant increase in BMI than the delayed group (OI and OD) respectively. This can be explained by the increase in muscle weight in the immediate group than the delayed one. Boualga et al. (24) found comparable results.
The present study demonstrated a statistically significant higher total weight of the retroperitoneal, epididymal and mesenteric fat in the obese groups than the non obese ones. This agreed with Pinheiro-Mulder et al. (20) Who found that retroperitoneal fat mass was significantly greater in obese group (diet-induced obesity) than in control group, also, the high-fat feeding used in this study led to dramatic increases in the total amount of adipose tissue (14).

In present study, gastrocnemius was chosen as it is intensively involved in swimming (22). The gastrocnemius muscle had a lower muscle weight in obese groups in relation to its corresponding control. This was agreed with Arnold et al. (16) and Paturi et al. (25) who explained that obesity decreased plasma levels of growth hormones and thyroid hormones this hinder skeletal ms growth and produced insulin resistance accelerated muscle degradation.

The trained immediately feeding rats (CI and OI) showed significantly higher muscle weight than the corresponding delayed feeding one (CD and OD) respectively. This agreed with previous researchers $(26,27)$. Leenders et al. (28) found that timing of protein intake after training is important for the development of hypertrophy in skeletal muscle as a result of larger net muscle protein synthesis in immediate training compared to the delayed one.

The effect of obesity on muscle glycogen content is controversial: in the present study muscle glycogen was slightly significant increase in the obese groups than the control ones. This was 


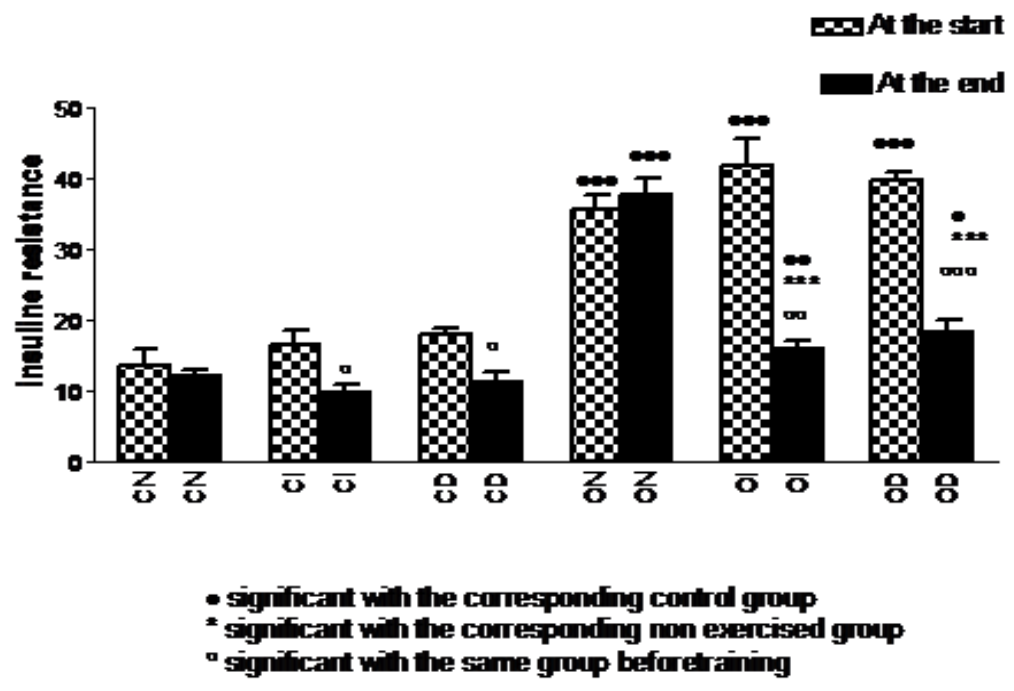

Fig. (7): Difference in insulin resistance between different groups at the beginning and the end of the study.

agreed with Elj, et al. (17). However, Goodpaster et al. (29) reported that obesity is associated with insulin resistance that is characterized by a reduced transport and/or phosphorylation of glucose and lower rates of muscle glycogen synthesis. The difference between different studies may result from using different exercise intensity because the high-intensity exercise leads to more rapid carbohydrate oxidation; whereas lowintensity as used in the present work bouts are beneficial for the consumption of fatty-acid fuel (30).

In the present study, the trained groups (CI and $\mathrm{CD}$ ) and (OI and OD) showed higher muscle glycogen than the sedentary one ( $\mathrm{CN}$ and $\mathrm{ON})$ respectively. This agreed with Luciano et al. (31). Training increases PI3-kinase activity (31). That enhances insulin sensitivity, glycogen synthase expression $(32,33)$. In this study, muscle glycogen was significantly higher in the immediate than the delayed feeding groups. This agreed with Cribb and Hayes (34).
The increased muscle mass produces an increase in the resting metabolic rate which could explain the decreased adipose tissue weight in the immediate versus the delayed feeding groups (35). In the present work, the immediate group showed insignificant difference in plasma glucose, insulin and insulin resistance between the immediate (CI and $\mathrm{OI}$ ) and delayed group (CD and $\mathrm{OD}$ ) respectively, this agreed with Tang et al. (36).

The high-fat diet used in our work significantly increase the plasma levels of glucose and insulin (17) that may be explained by the increased liver triacyl glycerol in association with maximal lipid storage capacity in visceral adipose tissue (14). The increased lipid availability is associated with diminished insulin-stimulated glucose uptake and glycogen synthesis that was proposed to account for the generation of lipid induced insulin resistance, ranging from substrate competition between lipids and carbohydrates to the activation of inhibitory signal transduction. That interferes with the insulin signaling pathways and stimulating glucose transport and glycogen synthesis (37). This sparing effect of carbohydrate 
substrate may explain the higher levels of glycemia and insulinemia with high fat diet (17).

Physical inactivity promotes obesity and insulin resistance, whereas physical activity and exercise reduce these risks (38). In the present study, the plasma glucose, insulin and insulin resistance were higher between sedentary ( $\mathrm{CN}$ and $\mathrm{ON})$ than trained (OI, OD, CI and $\mathrm{CD}$ ) groups. This is agreed with Morris et al. (39), who found that exercise reduced insulin and blood glucose levels. Also, Chen et al. (40), found that regular exercise improves glycemic control, as measured by glycated hemoglobin and fasting blood glucose levels, and ameliorates insulin resistance in obese groups. This could explained by Increased expression of hexokinase, translocation of glucose transporter 4 (GLUT4) to the cell surface and expressing higher levels of insulin receptors in the adipocytes (14).

Conclusion: Immediate post exercise feeding is better than three hours later as a strategy for glucose homeostasis.

\section{REFERENCES}

1- Steckling FM, Lima FD, Farinha JB, Dos Santos DL, Antunes Soares FA. Obesity, Inflammation and Aerobic Physical Exercise. Ann Sports Med Res 2(2): 1017, 2015.

2- Badran M. and Laher I.: Obesity in ArabicSpeaking Countries, Journal of Obesity, Article ID 686430, 9 pages, 2011.

3- Elamin A.: Epidemiologic and aetiopathological aspects. Khartoum Medical Journal, 03(3):457 - 465, 2010.

4- Knowler W., Barrett-Connor E., Fowler S., Hamman R., Lachin J., Walker E. and Nathan D.: Diabetes Prevention Program Research Group. Reduction in the incidence of type 2 diabetes with lifestyle intervention or metformin. N. Engl. J. Med., 346: 393-403, 2002.

5- Bertz F., Winkvist A. and Brekke H.: Sustainable weight loss among overweight and obese lactating women is achieved with an energy-reduced diet in line with dietary recommendations: results from the LEVA randomized controlled trial. J Acad Nutr Diet. 115(1):78-86, 2015.

6- Garaulet M, Gómez-Abellán. Timing of food intake and obesity: a novel association P.Physiol Behav. 2014; 134:44-50

7- Noeman S., Hamooda H., Baalash A.: Biochemical Study of Oxidative Stress Markers in the Liver, Kidney and Heart of High Fat Diet Induced Obesity in Rats. Diabetol. Metab. Syndr. 3,17. 2011.

8- Campos G., Volpato I., Calderon M., Rudge, Damasceno D: Effect of obesity on rat reproduction and on development of their adult offspring. K.E. Brazilian Journal of Medical and Biological Research. 41, 122125.2008

9- Estadella D., Oyama L., Damaso A., Ribeiro E., Oller D., Nascimento C. Effect of palatable hyperlipidic diet on lipid metabolism of sedentary and exercised rats. Nutrition. 20, 218-224, 2004.

10- Hwang DL, Barseghian G, Lev-Ran A: Determination of free-insulin in antibody containing sera. Horm Metab Res 17, 595-597, 1985. 
11- Tietz NW: Clinical guide to laboratory tests (3 rd ed), pp. 268-273, Philadelphia, WB Saunders, 1995.

12- Saad MF, Anderson R, Laws A: A comparison between the minimal model and the glucose clamp in the assessment of insulin sensitivity across the spectrum of glucose tolerance. Diabetes 43, 1114-1121, 1994

13- Kemp A. and Adrienne J: Determination of liver glycogen. Biochem. J., 65: 646. . 1954.

14- Gollisch K., Brandauer J., Jessen N., Toyoda N. and Goodyear H.: Effects of exercise training on subcutaneous and visceral adipose tissue in normal- and high-fat diet-fed rats. Am J Physiol Endocrinol Metab., 297(2): 495-504.2009

15- Abdel Malek H.: Chronic Exenatide administration and glucose \& lipid metabolism in lean and obese rats, International Journal of Biological \& Pharmaceutical Research, 38: 996-999, 2012.

16- Arnold C., Doyle J., Ingalls C., Rupp J., Reiser P., Kakarla S., Rice K. and Blough E.: Insulin resistance does not inhibit the ability of overload to induce hypertrophy in the obese Zucker rat Leprfa plantaris, Science \& Sports, 28(3): 133-139, 2012.

17- Elj N., Lacb G., Zaouali M., Tabka Z., Gharbi N. and El Fazaa S.: Interaction of high-fat versus high-carbohydrate diets and physical training on energy metabolism in the rat, Science \& Sports, 22:286-288, 2007.

18- Paula Pinto A, Massafera G, Jordao A, Costa T: Anthropometric and Biochemical Parameters of Rats Treated with a Lowcarbohydrate Diet Universal. Journal of Food and Nutrition Science, 1, 16 - 21. 2013.
19- Hariri $\mathbf{N}$ and Thibault L: High-fat dietinduced obesity in animal models. Nutrition Research Reviews, 23, 270-299. 2010

20- Pinheiro-Mulder A., Aguila M., Bregman R.and Mandarim-de-Lacerda C.: Exercise counters diet-induced obesity, proteinuria, and structural kidney alterations in rat. Pathol Res Pract., 206 (3):168-173, 2010.

21- Schroeder M., Shbiro L., Gelber V. and Weller. :Post-weaning voluntary exercise exerts long-term moderation of adiposity inb males but not in females in an animal model of early-onset obesity. Hom Behav., 574(5): 496$505,2010$.

22- Matsakas A., Bozzo C., Cacciani N., Caliaro F., Reggiani C., Mascarello F. and Patruno M.: Effect of swimming on myostatin expression in white and red gastrocnemius muscle and in cardiac muscle of rats. Exp Physiol., 91(6): 983-994, 2006.

23- Melanson E., Gozansky W., Barry D., MacLean P., Grunwald G. and Hill J. : When energy balance is maintained, exercise does not induce negative fat balance in lean sedentary, obese sedentary, or lean endurancetrained individuals. J Appl Physiol., 107:18471856, 2009.

24- Boualga A., Prost J., Taleb-Senouci D., Krouf D., Kharoubi O, Lamri-Senhadji M., Belleville J. and Bouchenak M.: Purified chickpea or lentil proteins impair VLDL metabolism and lipoprotein lipase activity in epididymal fat, but not in muscle, compared to casein, in growing rats. Eur J Nutr. 48(3):1629, 2009. 
25- Paturi S., Gutta A., Kakarla S., Katta A., Arnold E. and Wu M.: Impaired overloadinduced hypertrophy in obese Zucker rats lowtwitch skeletal muscle. J Appl Physiol., 1081:7-13, 2010.

26- Hartman J., Tang J., Wilkinson S., Tarnopolsky M., Lawrence R, Fullerton A. and Phillips S.: Consumption of fat-free fluid milk after resistance exercise promotes greater lean mass accretion than does consumption of soy or carbohydrate in young, novice, male weightlifters. Am J Clin Nutr., 86:373-381, 2007.

27- Poole C., Wilborn C., Taylor L. and Kerksick C.: The role of post-exercise nutrient administration on muscle protein synthesis and glycogen synthesis, Journal of Sports Science and Medicine, 9: 354 - 363, 2010.

28- Leenders M., Verdijk L.,Van d.,Van K., Nilwik R., Wodzig W., Senden J., Keizer H. and Van L.: Protein supplementation during resistance-type exercise training in the elderly. Med Sci Sports Exerc. 45(3):542-52, 2013

29- Goodpaster B.; Wolfe R. and Kelley D.: Effects of Obesity on Substrate Utilization during Exercise, Obesity research, 10(7):57584. 2002

30- Egan B. and Zierath J.: Exercise Metabolism and the Molecular Regulation of Skeletal Muscle Adaptation, Cell Metabolism, 17: 162184. 2013.

31- Luciano E., Carneiro E., Carvalho C., Carvalheira J., Peres S., Reis M., Saad M., Boschero A. and Velloso L.: Endurance training improves responsiveness to insulin and modulates insulin signal transduction through the phosphatidylinositol 3-kinase/Akt1 pathway, European Journal of Endocrinology, 147(1):149-157, 2002.

32- Wojtaszewski J., Hansen B. and Gade E.: Insulin signaling and insulin sensitivity after exercise in human skeletal muscle. Diabetes, 49(3):325-331, 2000.

33- Manabe Y., Gollisch K., Holton L., Kim Y., Brandauer J., Fujii N., Schroeder M., Shbiro L., Gelber V. and Weller A.: Hormones and Behavior, 57(4-5): 496-505, 2010.

34- Cribb P. and Hayes A.: Effects of supplement timing and resistance exercise on skeletal muscle hypertrophy. Med Sci Sports Exerc., 38:1918-1925, 2006.

35- Al-Barazanji A., McNulty J., Binz J., Generaux C., Benson W., Young A. and Chen L.:Synergistic effects of a GPR119 agonist with metformin on weight loss in DIO mice. Pharmacol Exp Ther. pii: jpet.115.222828, 2015.

36- Tang J., Perco J., Moore D., Wilkinson S. and Phillips S.: Resistance training alters the of response fed state mixed muscle protein synthesis in young men. Am J Physiol Regul Integr Comp Physiol. 294(1):R172-8, 2008.

37- Taylor A., Ye1 J. and Schmitz-Peiffer C.: Inhibition of glycogen synthesis by increased lipid availability is associated with subcellular redistribution of glycogen synthase, Journal of Endocrinology, 188: 11-23, 2006.

38- Weiss R. and Kaufman. : Metabolic Complications of Childhood, Obesity, and Diabetes Care 31 Suppl., 2:S310-S316, 2008. 
39- Morris R., Laye M., Lees S., Rector R., Thyfault J. and Booth F.: Exercise-induced attenuation of obesity, hyperinsulinemia, and skeletal muscle lipid peroxidation in the Oletf rat. J, Appl.physiol., 104:708-715, 2008.

40- Chen L., Pei J., Kuang J., Chen H., Chen Z., Li Z. and Yang Z.: Effect of lifestyle intervention in patients with type 2 diabetes: A meta-analysis. Metabolism, 64 (2): 338-347, 2015. 